Pratiques et usages de l'écrit diplomatique à SaintFlorent de Saumur (ca 950-1203)

Thèse de doctorat, université d'Angers, sous la direction de Jean-Michel Matz, soutenue le 20 décembre 2018

Paul-Henri Lécuyer

OpenEdition
Journals
Édition électronique
URL : https://journals.openedition.org/cem/16548
DOI : $10.4000 /$ cem. 16548
ISSN : $1954-3093$
Éditeur
Centre d'études médiévales Saint-Germain d'Auxerre

Référence électronique

Paul-Henri Lécuyer, «Pratiques et usages de l'écrit diplomatique à Saint-Florent de Saumur (ca 950-1203) », Bulletin du centre d'études médiévales d'Auxerre | BUCEMA [En ligne], 23.1 | 2019, mis en ligne le 01 septembre 2019, consulté le 22 septembre 2022. URL : http://journals.openedition.org/ cem/16548; DOI : https://doi.org/10.4000/cem.16548

Ce document a été généré automatiquement le 22 septembre 2022.

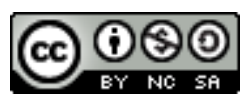

Creative Commons - Attribution - Pas d'Utilisation Commerciale - Partage dans les Mêmes Conditions 4.0 International - CC BY-NC-SA 4.0

https://creativecommons.org/licenses/by-nc-sa/4.0/ 


\section{Pratiques et usages de l'écrit diplomatique à Saint-Florent de Saumur (ca 950-1203)}

Thèse de doctorat, université d'Angers, sous la direction de Jean-Michel Matz, soutenue le 20 décembre 2018

\section{Paul-Henri Lécuyer}

1 La thèse de doctorat que nous avons réalisée s'inscrit dans un courant d'étude très prisé par les historiens médiévistes depuis environ un demi-siècle: la question de l'écrit au Moyen Âge qui a donné matière à de multiples travaux. Il a également amené d'importantes remises en question, creusé de nouvelles pistes de recherches et redéfini les relations entre l'histoire et les sciences dites "auxiliaires ", notamment la diplomatique, à la faveur du renouvellement profond de ses principes fondamentaux - hérités pour une bonne partie de la méthodologie élaborée par dom Mabillon et développée par l'érudition historique du $\mathrm{XIX}^{\mathrm{e}}$ siècle - au cours de la seconde moitié du $\mathrm{xx}^{\mathrm{e}}$ siècle ${ }^{1}$,

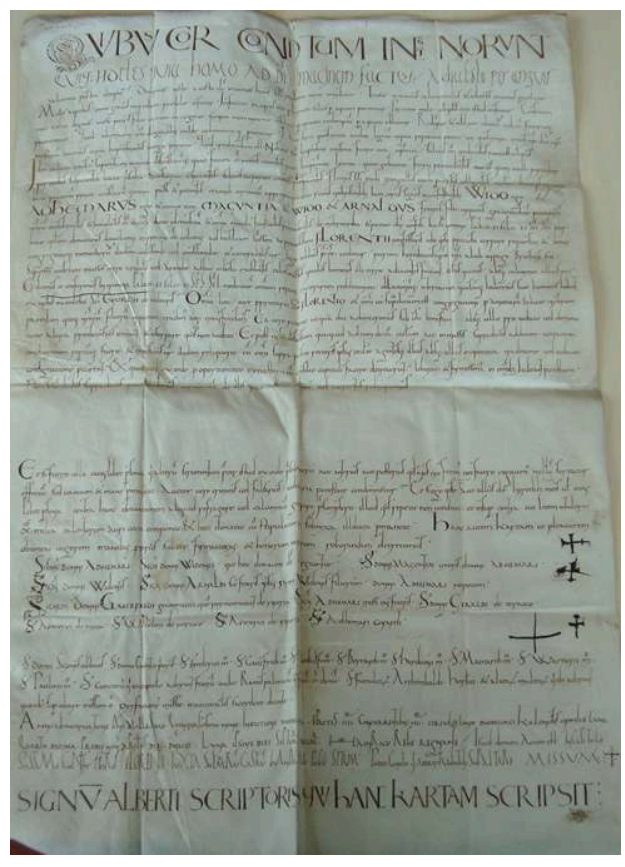
et des travaux sur la culture de l'écrit autour de la thématique de la Literacy $^{2}$.

2 Pour explorer ce thème, nous avons choisi comme sujet de recherche l'abbaye SaintFlorent de Saumur, qui, par la richesse exceptionnelle de son fonds d'archives ${ }^{3}$, constitue un terrain fécond pour l'étude de l'écrit au Moyen Âge. La date de 950, 
correspondant à l'installation de moines au castrum de Saumur par l'entremise du comte de Blois Thibaud $\mathrm{I}^{\text {er }}$ le Tricheur ${ }^{4}$, a été retenue comme point de départ de l'étude qui porte sur les pratiques et usages de l'écrit diplomatique des frères de l'abbaye de Saint-Florent. Pour ce faire, la foisonnante production documentaire a été examinée en profondeur afin de reconnaître ses spécificités propres, tant du point de vue de la forme que du contenu des actes, et de voir comment les religieux géraient ces flux documentaires. L'enjeu majeur était de mieux comprendre les implications de l'acte d'écrire au sein de cet établissement en évaluant le rôle qu'ont pu tenir ces actes, à la fois dans le fonctionnement "quotidien» de l'abbaye, dans l'affermissement de son pouvoir temporel et dans le processus de construction et d'affirmation de son identité. Nous avons retenu comme terminus ad quem l'année 1203, qui renvoie à la fin de l'abbatiat de Mainier (commencé en 1176), l'un des derniers grands abbés de SaintFlorent, qui ont construit la puissance de l'abbaye saumuroise au cours des $\mathrm{XI}^{\mathrm{e}}$ et XII siècles. L'intitulé de notre travail de thèse ainsi que les bornes chronologiques, que nous avons définies, expriment clairement l'objet de cette étude et les choix que nous avons effectués pour délimiter le périmètre de notre corpus ${ }^{5}$. Ainsi, la question des " pratiques » et des " usages » de l'écrit diplomatique a été traitée sous divers aspects complémentaires, qui ont été regroupés en quatre chapitres.

Le premier chapitre fait office de prolégomènes à cette enquête sur l'écrit "florentin " et offre une vue d'ensemble de notre corpus, que nous avons soumis à un triple questionnement. Tout d'abord, nous avons effectué une analyse chronologique des unités documentaires étudiées ${ }^{6}-1123$ en tout, dont 278 sont des doublons ${ }^{7}-$, qui a mis en lumière une forte concentration de la production documentaire dans la seconde moitié du xi ${ }^{e}$ siècle, en particulier durant les deux dernières décennies ${ }^{8}$, puis une baisse au cours du $\mathrm{XII}^{\mathrm{e}}$ siècle$^{9}$. Ensuite, nous avons procédé à une analyse géographique de notre corpus en scrutant la répartition des unités documentaires en fonction des dépendances auxquelles elles se rapportent. Elle a fait ressortir la présence des moines de Saint-Florent dans vingt et un diocèses français ${ }^{10}$, ainsi qu'en Angleterre, et a permis de retracer les grandes lignes du processus d'extension de la congrégation florentine dans une large zone, allant de la Gascogne aux marches des Pays de Galles et de l'estuaire de la Rance à la région de Gien, en passant par l'île-de-France. Enfin, nous avons traité de la typologie des actes en identifiant quatre grandes catégories diplomatiques: en premier lieu, les chartes, ainsi que les notices - qui représentent respectivement $37 \%$ et $57 \%$ de notre documentation -, mais aussi les actes pontificaux (bulles et mandements) et, de façon beaucoup plus limitée, les relevés de propriété censiers, relevés de tenures, listes d'églises relevant d'un prieuré donné. À côté des notices, auxquelles les moines ont eu largement recours, tout particulièrement entre 1061 et $1100^{11}$, nous avons répertorié 352 actes comportant un ou plusieurs auteur(s) nommément désigné(s), qu'il s'agisse de personnes physiques ou d'institutions. L'examen de ces auteurs d'actes, en majorité ecclésiastiques $(58 \%)^{12}$, laisse entrevoir toute la gamme des catégories sociales avec lesquelles les moines pouvaient entrer en relation, notamment comme bénéficiaires d'aumônes de diverses natures ou en tant qu'adversaires dans le cadre de conflits de propriété ou de contestations de droits.

Dans le deuxième chapitre, nous avons porté notre attention sur la forme de ces documents, à la fois sous l'angle de leurs caractères externes - en nous penchant sur le support, les dimensions, le format, la mise en page et les caractéristiques d'écriture des 
actes sur parchemin - et sous celui des caractères internes, à travers l'analyse des parties du discours diplomatique et des signes de validation - souscriptions et scellement des actes -, qui assurent la valeur juridique de l'acte et servent parfois à l'authentifier. En ce qui concerne l'aspect matériel des documents, on observe ainsi dans plusieurs pièces du chartrier, surtout dans la seconde moitié du XI siècle, un véritable souci apporté à la présentation formelle de l'acte, qui se matérialise par un support de qualité, une mise en page et une écriture soignées ou la présence d'éléments ornementaux et/ou symboliques d'une grande diversité. Ainsi, le traitement des invocations symboliques, et spécialement de celles que Chantal Senséby a qualifiées de type "fleuri », paraît constituer une véritable "marque de fabrique» du centre d'écriture de Saint-Florent ${ }^{13}$. De même, nous avons identifié un certain nombre de préambules longs et de trames récurrentes semblant bien provenir du même scriptorium. Nous avons également souligné l'importance juridique et symbolique que revêtait le processus de validation des actes et relevé différentes informations contenues dans la corroboration et le protocole final apportant d'utiles éléments pour comprendre la genèse des actes ${ }^{14}$.

5 Le troisième chapitre aborde la question de la transmission et de la réception de l'écrit en étudiant, tout d'abord, le phénomène de la copie sous trois formes - sur parchemin isolé, pancarte ou en rouleau -, dans le sillage des travaux ayant renouvelé l'approche des phénomènes de tradition ${ }^{15}$, qui ne sont désormais plus réduits à de simples miroirs déformants d'un original qui ferait office de référence absolue. Ces trois formes documentaires n'avaient pas vocation à concerner exactement les mêmes types d'actes. Ainsi, les rouleaux ${ }^{16}$, mais également les exemplaria ${ }^{17}$, comportent plus volontiers des actes émanant d'un auteur prestigieux, y compris des documents suspects voire faux, tandis que les pancartes consignent davantage des documents relatifs à des transactions de modeste importance ${ }^{18}$. À cette étude des états de tradition des actes, nous avons choisi d'adjoindre celle de leur réception, y compris la manière avec laquelle les documents sont compris, annotés, classés, puis utilisés par les religieux florentins. Concrètement, nous nous sommes focalisés sur certains aspects du devenir de l'écrit après sa création, en premier lieu sur les notes dorsales que l'on trouve sur l'écrasante majorité des actes sur parchemin du corpus et qui permettaient, grâce aux informations qu'elles fournissent ${ }^{19}$, de faciliter leur classement. Ensuite, nous nous sommes intéressés aux utilisations spécifiques de l'écrit, tout spécialement dans sa dimension juridique, en nous concentrant sur la pratique du chirographe qui contribua utilement au cours du XII e siècle à affirmer le rôle probatoire de l'écrit à Saint-Florent de Saumur, en fixant à la fois son contenu textuel et sa forme externe ${ }^{20}$. Parallèlement au développement de cet instrument, on assiste, à partir de la fin $\mathrm{du} \mathrm{XI}^{\mathrm{e}}$ siècle, et surtout au cours $d u \mathrm{XII}^{\mathrm{e}}$ siècle, à un recours croissant à l'écrit sous toutes ses formes dans les procédures de gestion des conflits et, en particulier, à l'occasion des plaids, et à un souci plus marqué de conserver par écrit - notamment par le biais de notices - les faits judiciaires, y compris les simples arrangements privés.

6 Le quatrième et dernier chapitre consiste en une étude comparée des cartulaires de Saint-Florent - le Livre noir, le Livre blanc et le Livre d'argent, réalisés au $\mathrm{XI}^{\mathrm{e}}$ et au $\mathrm{XII}^{\mathrm{e}}$ siècle -, en procédant pour chaque recueil à une description matérielle du codex, à un examen approfondi de son contenu, puis de sa structure interne en analysant l'ordre de classement des unités documentaires adopté par les cartularistes. Cette analyse conjointe des trois manuscrits nous a permis de mieux comprendre leurs modalités 
d'élaboration, leurs caractéristiques respectives, ainsi que leur raison d'être. Ainsi, on se rend compte que les critères de sélection des documents à copier n'étaient pas uniformes, qu'ils ont connu des évolutions entre l'époque de rédaction du Livre noir sous l'abbatiat de Sigon (1055-1070) et celle qui a vu la réalisation des Livres blanc et d'argent, à la charnière des années 1170 et 1180 , et qu'ils pouvaient fluctuer au sein d'un même recueil. La démarche intellectuelle qu'implique la confection d'un cartulaire se révèle, notamment, dans l'agencement du classement des actes, qui apparaît plus ou moins cohérent dans les trois manuscrits analysés ${ }^{21}$ et obéit à des logiques différentes. En ce qui concerne leur contenu, outre les divergences relatives à la chronologie des unités documentaires transcrites dans les trois cartulaires, nous avons mis en évidence des choix différenciés mis en œuvre par leurs concepteurs respectifs : ces choix portent sur la nature des actes copiés, mais également sur les domaines et dépendances concernés, révélant, notamment, une certaine complémentarité en matière de répartition géographique des transcriptions entre le Livre blanc et le Livre d'argent. Tout ceci a permis d'entrevoir, avec plus de précision, les préoccupations des commanditaires des cartulaires de Saint-Florent et, par là même, des fonctions qui pouvaient leur être assignées, à dominante plutôt gestionnaire et juridique pour le Livre blanc, tandis que le Livre noir et le Livre d'argent ont une tonalité mémorielle plus évidente. Le Codex niger comporte même des insertions de textes de nature historiographique qui se marient subtilement avec les écrits diplomatiques et visent à rappeler l'ancienneté de l'abbaye de Saint-Florent, en enracinant son origine dans les temps carolingiens, perçus aux $\mathrm{XI}^{\mathrm{e}}-\mathrm{XII}^{\mathrm{e}}$ siècles comme une période de référence ${ }^{22}$.

7 Au terme de ce travail d'analyse de l'écrit diplomatique à Saint-Florent de Saumur et en guise de prolongement de cette thèse, il serait intéressant de mesurer ce qui relève vraiment des caractéristiques intrinsèques du scriptorium florentin, en comparant sa production avec celle des institutions religieuses du Val de Loire, en élargissant éventuellement aux régions voisines (Poitou, Bretagne, Maine), y compris en incluant dans l'enquête les fonds épiscopaux. Cela permettrait de mieux cerner la perméabilité des pratiques à l'échelle régionale prise au sens large, la circulation des influences et, peut-être, la part prise par l'abbaye de Saint-Florent dans la diffusion de modèles scripturaires.

Reçu : 27 février 2019 - Accepté : 14 juin 2019

\section{NOTES}

1. C'est essentiellement à compter des années 1950 que s'opère à cet égard une inflexion majeure, sous l'influence du médiéviste autrichien Heinrich Fichtenau : le document n'est plus seulement considéré à l'aune des informations qu'il contient et les parties du discours diplomatique font dès lors l'objet d'une attention plus poussée, y compris dans leur aspect récurrent ou supposément superfétatoire. Non contents de dépoussiérer les méthodes d'analyse des caractères internes et externes, les historiens et diplomatistes ont ensuite cherché à élargir le spectre de leurs secteurs de recherche en s'occupant du contexte entourant le processus d'élaboration de l'acte (sa "genèse »), puis de l'état dans lequel il a été transmis à la postérité, 
autrement dit la «tradition ", et, enfin, à la réception des documents, ce qui a amené à prendre davantage en compte leur matérialité.

2. Ces approches ont en grande partie été influencées par les recherches effectuées par l'anthropologie sociale britannique et, en premier lieu, par les travaux de Jack Goody : J. Goody et I. WATT, "The consequences of literacy ", Comparative studies in society and history, 5/3 (1963), p. 304-345 ; ID. (éd.), Literacy in traditional societies, Cambridge, 1968. Citons également l'ouvrage fondamental de Michael Clanchy, qui a remis à l'honneur l'apport du Moyen Âge dans la culture occidentale de l'écrit: M. CLANCHY, From Memory to Written Record. England, 1066-1307, Londres, 1979. Des éditions revues et augmentées ont été publiées en 1993 et 2013. L'historiographie française est, quant à elle, restée plutôt en retrait de ce courant autour de la Literacy. Elle s'est néanmoins approprié les questionnements portants sur la part prise par l'écrit dans la formation des communautés médiévales, sous l'impulsion de la thèse de doctorat d'État de Michel Zimmermann, soutenue en 1992 et publiée en 2003 : M. ZIMMERMANN, Écrire et lire en Catalogne du $I^{e}$ au XII siècle, 2 vol., Madrid, 2003. Explorant les pistes ainsi tracées, un certain nombre de travaux universitaires a été réalisé à partir des années 2000, en ayant pour fil conducteur les pratiques de l'écriture des institutions médiévales - en premier lieu des communautés monastiques - et/ou de la contribution essentielle de l'écrit dans l'élaboration et la conservation de la memoria de celles-ci. On citera, notamment, les thèses de doctorat de Marlène Hélias-Baron (Recherches sur la diplomatique cistercienne au XII siècle: La Ferté, Pontigny, Clairvaux, Morimond, université Paris 1 Panthéon-Sorbonne, dir. Michel Parisse, 2005), de Dominique Stutzmann (Écrire à Fontenay: esprit cistercien et pratiques de l'écrit en Bourgogne, XII ${ }^{e}-\mathrm{XIII}^{e}$ siècles, université Paris 1 Panthéon-Sorbonne, dir. Michel Parisse, 2009), de Claire Garault (Écriture, histoire et identité : la production écrite monastique et épiscopale à Saint-Sauveur de Redon, Saint-Magloire de Léhon, Dol et Alet/ Saint-Malo, milieu du IX ${ }^{e}$ siècle-milieu du XII ${ }^{e}$ siècle, université de Rennes 2, dir. Bernard Merdrignac, 2011) ou de Jean-Baptiste Renault (L'écrit diplomatique à Saint-Victor de Marseille et en Provence, ca. 950-ca. 1120, université de Strasbourg, dir. Benoît-Michel Tock, 2013).

3. Tel qu'il se présente actuellement, le chartrier de l'abbaye de Saint-Florent regroupe des pièces du IX $\mathrm{X}^{\mathrm{e}}$ au XVIII ${ }^{\mathrm{e}}$ siècle - ainsi que des notes d'érudits des $\mathrm{XIX}^{\mathrm{e}}$ et $\mathrm{XX}^{\mathrm{e}}$ siècles -, intéressant à la fois l'ancien monastère du Mont-Glonne, l'abbaye Saint-Florent de Saumur proprement dite et ses nombreux prieurés. Il est dans sa quasi-totalité conservé aux Archives départementales de Maine-et-Loire, au sein desquels il occupe plus de 82 mètres linéaires. En outre, il existe de nombreuses copies de chartes de l'abbaye de Saint-Florent - essentiellement des copies de l'époque moderne ou du XIX ${ }^{\mathrm{e}}$ siècle - dans des dépôts extérieurs au département, en particulier aux Archives nationales et à la Bibliothèque nationale de France, où l'on trouve, notamment, dans les nouvelles acquisitions latines (nouv. acq. lat. 1930), le plus ancien cartulaire de SaintFlorent (le Livre noir), qui fait partie de notre corpus de sources.

4. Dès le haut Moyen Âge, une communauté de moines de Saint-Florent était implantée sur le site du Mont-Glonne à Saint-Florent-le-Vieil. Chassés de leur monastère par les Normands en 853, les religieux se retirèrent jusqu'à Tournus, en Bourgogne.

5. Nous n'y avons pas inclus les actes originaux antérieurs à 950, ainsi que les actes transcrits dans le Livre rouge, qui, bien que bon nombre d'entre eux soient datés du XII siècle, figurent dans un codex rédigé au XIII ${ }^{\mathrm{e}}$ siècle. De même, du fait de l'attention particulière que nous accordons à la matérialité des documents, nous n'avons pas pris en compte les copies d'actes des $\mathrm{X}^{\mathrm{e}}, \mathrm{XI}^{\mathrm{e}}$ ou XII ${ }^{\mathrm{e}}$ siècles réalisées après 1203, que l'on trouve en nombre important dans les archives de l'abbaye Saint-Florent de Saumur et qui ont été effectuées pour la plupart durant l'époque moderne. Par ailleurs, nous avons pris le parti d'écarter les sources narratives relatives à l'établissement saumurois et de resserrer notre champ de recherche sur l'exceptionnel gisement d'actes de la pratique florentins, sans s'interdire le recours ponctuel à deux textes de nature 
historiographique copiés dans le Livre noir, et en ayant ponctuellement recours aux sources narratives, en particulier l'Historia Sancti Florentii Salmurensis, pour illustrer tel ou tel point.

6. Nous avons ici utilisé de préférence le terme générique d'« unité documentaire " à celui d'« acte ", dans la mesure où il recouvre à la fois les chartes, les notices, les actes pontificaux (bulles et mandements), mais également les écrits non diplomatiques compris dans notre corpus. 7. Au total, 331 unités documentaires sont parvenues jusqu'à nous sous forme de documents (originaux et doublons) sur parchemin ( $29 \%$ du total), 33 l'ont été sous forme de transcriptions dans un rouleau et 759 nous sont connues par les trois cartulaires compris dans le périmètre de notre étude (Livre noir, Livre blanc et Livre d'argent), qui constituent le mode de transcription prépondérant (68\%) des actes de l'abbaye Saint-Florent de Saumur entre la seconde moitié du $\mathrm{x}^{\mathrm{e}}$ et le tout début du XIII ${ }^{\mathrm{e}}$ siècle.

8. La seconde moitié $\mathrm{du} \mathrm{xI}^{\mathrm{e}}$ siècle connaît une augmentation continue de la production diplomatique florentine : on compte ainsi 89 unités documentaires entre 1041 et 1060, 169 pour la période 1061-1080 et pas moins de 233 unités documentaires entre 1081 et 1100 . Le long abbatiat du successeur de Sigon, Guillaume de Dol (1070-1118), concentre à lui seul une proportion d'environ $43 \%$ du total des unités documentaires du corpus étudié.

9. Concrètement, on dénombre 79 unités documentaires entre 1101 et 1120,49 durant la période 1121-1140, 80 en 1141-1160, 49 en 1161-1180 et 31 entre 1181 et 1203.

10. Les zones d'implantation les plus importantes sont les diocèses d'Angers (plus de $38 \%$ des unités documentaires concernent l'abbaye mère ou des dépendances angevines) et de Poitiers (18\% des unités documentaires).

11. Au total, 484 notices ont été comptabilisées pour notre période d'étude, dont 169 datent de la tranche chronologique 1061-1080 et 233 de 1081-1100. Certaines de ces notices sont très brèves et présentent un formulaire minimaliste, alors que d'autres se caractérisent par une narration parfois très riche et mobilisant une rhétorique foisonnante, dans le but de conserver la mémoire de certains faits, notamment dans le cadre des conflits judiciaires.

12. Quarante et un actes ont pour auteur un pape, quinze émanent de légats ou de commissaires pontificaux, trente-huit de rois et princes territoriaux, quatre-vingt-quatorze d'évêques, six de chapitre cathédraux, de doyens ou d'archidiacres, cent dix de seigneurs et autres laïcs, quatre de moines ou de prêtres. Enfin, quarante-deux chartes ont pour auteur un abbé, un moine ou la communauté des moines de Saint-Florent de Saumur et deux émanent d'autres abbés et communautés religieuses.

13. C. S ENSÉBY, «Les invocations symboliques: diversité graphique, fonction identitaire et circulation des modèles (espace ligérien, $\mathrm{x}^{\mathrm{e}}-\mathrm{XII}^{\mathrm{e}}$ siècle)", in C. SENSÉBY, L'écrit monastique dans l'espace ligérien ( $\mathrm{X}^{e}$-XIII ${ }^{e}$ siècle). Singularités, interférences et transferts documentaires, Rennes, 2018, p. 130-131. L'auteur décrit ce type graphique en ces termes : « Il affiche à son sommet un vestige ou un embryon de boucle sommitale demeurée ouverte, arbore une série de trois ou quatre clés de sol, une double armature, une pointe finale et, parsemant le tout, des points distribués en triangle (Amen ?) ou de petits signes en forme de "v" parfois pointés. »

14. Si l'on considère les dates de lieu des documents, il apparaît que le fait qu'un acte ait été établi à l'abbaye de Saint-Florent de Saumur ou dans un prieuré est un indice intéressant pour en attribuer la rédaction aux moines florentins.

15. Voir, notamment, les articles suivants: O. G UYOTJEANNIN et L. M ORELLE, "Tradition et réception de l'acte médiéval: jalons pour un bilan des recherches», Archiv für diplomatik, Schriftgeschichte, Siegel- und Wappenkunde, 53 (2007), p. 367-403, et L. MORELLE, « Usages et gestion de l'écrit documentaire (Occident, $\mathrm{VI}^{\mathrm{e}}-\mathrm{XII}^{\mathrm{e}}$ siècle) : quelques considérations sur l'acte écrit ", in L'autorité de l'écrit au Moyen Âge (Orient-Occident), Paris, 2009, p. 117-126.

16. Ces rouleaux sont au nombre de trois : le « rouleau des privilèges » (Archives dép. de Maineet-Loire, $\mathrm{H}$ 1836), qui a probablement été réalisé au début de la seconde moitié $\mathrm{du} \mathrm{XI}^{\mathrm{e}}$ siècle et 
rassemble des copies de cinq diplômes carolingiens et de la bulle suspecte du pape Jean XVIII, un rouleau de la fin du $\mathrm{XI}^{\mathrm{e}}$ siècle qui regroupe des actes concernant le prieuré de l'Abbaye-sous-Dol (Archives dép. de Maine-et-Loire, $\mathrm{H}$ 3331) et un rouleau opistographe du XII ${ }^{\mathrm{e}}$ siècle, qui comprend d'un côté des actes relatifs à des possessions de l'abbaye de Saint-Florent en Touraine (Archives dép. de Maine-et-Loire, $\mathrm{H}$ 1838) et, de l'autre, des unités documentaires se rapportant aux prieurés anglais de Monmouth et d'Andover (Archives dép. de Maine-et-Loire, H 3711).

17. D'après Laurent Morelle les exemplaria sont des copies isolées sur feuilles de parchemin, cf. L. MORELLE, « Instrumentation et travail de l'acte : quelques réflexions sur l'écrit diplomatique en milieu monastique au xI ${ }^{\mathrm{e}}$ siècle », Médiévales, 56 (2009), en ligne [http://journals.openedition.org/ medievales/5537], p. 16.

18. Nous avons choisi de considérer comme pancarte tout document regroupant sur un même feuillet de parchemin les transcriptions de plusieurs unités documentaires distinctes. Cette définition est reprise d'I. VÉRITÉ, «Des pancartes dans les fonds des prieurés de Marmoutier? L'exemple des prieurés poitevins", in M. PARISSE, P. PÉGEOT et B.-M. TOCK (dir.), Pancartes monastiques des $\mathrm{XI}^{e}$ et XII ${ }^{e}$ siècles, Turnhout, 1998, p. 63-95, à la p. 66. Dans le cadre de notre période d'étude, nous avons répertorié un total de trente-cinq pancartes.

19. Elles sont majoritairement de nature topographique, mais l'on trouve aussi des indications anthroponymiques, sur la nature des biens concernés par l'action juridique ou même des analyses.

20. Le corpus comprend en tout trente-deux actes délivrés sous une forme chirographaire.

21. On remarque, notamment, un fort contraste entre le Livre noir qui présente une structure assez irrégulière, laissant transparaître un certain empirisme, et le Livre blanc qui se caractérise par un classement définissant des sections géographiques clairement individualisées et, pour la plupart, bien ordonnées en interne.

22. Le premier de ces deux documents est un texte poétique relatant des faits mythifiés (récit de la destruction du Mont-Glonne par les Bretons), les Versiculi de eversione monasterii Sancti Florentii, qui figure au début du cartulaire (PARIS, Bibliothèque nationale de France, nouv. acq. lat. 1930, Livre noir, fol. $\left.6 \mathrm{r}^{\circ}-8 \mathrm{v}^{\circ}\right)$; le second est inséré plus loin dans le recueil (fol. $\left.82 \mathrm{v}^{\circ}-83 \mathrm{v}^{\circ}\right)$ et consiste en une liste des abbés défunts de Saint-Florent.

\section{INDEX}

Mots-clés : chartes, cartulaire, diplomatique, Literacy, pratiques de l'écrit, Saint-Florent de Saumur, usages de l'écrit

\section{AUTEUR}

\section{PAUL-HENRI LÉCUYER}

Chargé d'études documentaires aux Archives départementales de Maine-et-Loire 